\title{
Construcción de Intereses Vocacionales de Estudiantes que Participan de un Programa para Continuar Estudios de Pedagogía
}

Caroll A. Schilling ${ }^{(1,2)^{\star}}$, Karen P. Gajardo-Asbún ${ }^{(2)}$ y Lorena E. Alaluf(2)

(1) Centro de Investigación en Educación para la Justicia Social Universidad Católica del Maule (CIEJUS), Talca - Chile

(2) Universidad Católica del Maule, Talca - Chile. (cschilling@ucm.cl; kpgajardo@ucm.cl; alalufg@ucm.cl)

Recibido Ene. 21, 2019; Aceptado Mar. 18, 2019; Versión final May. 7, 2019, Publicado Oct. 2019

\section{Resumen}

Se analiza y discute sobre la construcción de intereses vocacionales de jóvenes que participan de un programa de preparación para continuar estudios de pedagogía en la Universidad Católica del Maule, Chile. Este estudio contempló un diseño mixto explicativo secuencial. Se aplicó un pre y post test vocacional a los estudiantes que ingresaron al Programa de Atracción de Talentos en Pedagogía durante el año 2017 y se desarrollaron entrevistas semiestructuradas con aquellos estudiantes que presentaron mayores variaciones en los resultados del test. Entre los factores que fortalecen el interés vocacional los estudiantes señalan desafíos formativos del programa, con especial valoración de las experiencias formativas que los conectan con referentes sociales significativos como son los docentes con trayectorias profesionales destacadas. Se concluye que la participación de estudiantes en programas de preparación para acceder a pedagogías contribuye a fortalecer la decisión de optar por la docencia.

\section{Construction of Vocational Interests of Students who Participate in a Program to Continue Pedagogy Studies}

\begin{abstract}
The construction of vocational interests of young people who participate in a preparation program to continue studies of pedagogy at the Universidad Católica del Maule, Chile are discussed and analyzed. The research uses a mixed sequential exploratory design. From a sequential explanatory mixed perspective, a battery of vocational tests was applied to the students entering the Talent Attraction Program in Pedagogy (APP) during the year 2017, and semi-structured interviews were applied to the students who presented greater variations in the test results. Among the factors that strengthen the vocational interest are the training challenges of the program, with special appreciation of the training experiences that connect them with significant social referents such as teachers with outstanding professional careers. It is concluded that the participation of students in preparation programs to access pedagogies, contributes to strengthen the decision to opt for teaching.
\end{abstract}




\section{INTRODUCCIÓN}

La atracción y sostenibilidad de los estudiantes a las carreras de pedagogía ha sido un ámbito de interés internacional. Entre los países con más alto desempeño en resultados de aprendizaje escolar destacan Singapur, Finlandia, Hong Kong y Corea del Sur, a través de la implementación de mecanismos de acceso a la docencia de estudiantes talentosos. Entre los procedimientos de detección y selección destacan Singapur y Finlandia como los más eficientes (Barber y Mourshed, 2008). Ambos sistemas ponen fuerte énfasis en los logros académicos de los postulantes, sus habilidades de comunicación y su motivación hacia la docencia. En América Latina, la formación de profesores enfrenta el desafío de atraer el mayor número de candidatos calificados para el ejercicio de la docencia (Said-Hung et al., 2017). Particularmente en el caso de Chile, se ha optado por seguir la tendencia mundial que presentan los sistemas de alto desempeño educativo, dado que tal como advierte la OCDE (2017) se requiere mejorar los niveles de equidad y el acceso a la educación superior. Asimismo, debieran tomarse medidas para reforzar el reclutamiento y los procesos de admisión en las instituciones universitarias, con el fin de apoyar a los estudiantes procedentes de entornos desfavorables para que puedan tener éxito durante sus estudios. Al respecto, Avalos (2014) y Vaillant (2016) evidencian que la formación docente presenta condiciones problemáticas respecto al perfil de los estudiantes que ingresan a estudiar pedagogía. En general, se identifican como los más pobres entre los estudiantes universitarios, con baja formación heredada de una educación secundaria deficiente y, en su mayoría, son primera generación de sus familias que acceden a estudios universitarios.

A partir de la Ley $n^{\circ} 20903$, promulgada el año 2016 en Chile, se crea el Sistema de Desarrollo Docente, el cual establece que las instituciones formadoras de profesores deben tener requisitos académicos de selectividad para cursar una carrera de pedagogía (Mineduc 2016). En este marco, las instituciones universitarias han intencionado estrategias que buscan atraer a estudiantes con buenos resultados académicos en la enseñanza secundaria e interés por estudiar pedagogía. Como señala González-García (2018), el proceso en el cual se identifica a un buen candidato a la pedagogía se inicia, en primer lugar, con "reconocer la subjetividad de un o una joven que manifiesta interés por la carrera pedagógica, como parte de su proyecto individual de autorrealización personal". Los propedéuticos pedagógicos en las universidades chilenas constituyen dispositivos de selección que abren espacios de formación, acercamiento del estudiante a la vida universitaria y, específicamente, buscan afianzar el interés por estudiar pedagogía, pues reconocen que "la labor de atraer a los mejores al ejercicio de la docencia, debería iniciarse antes del ingreso a la formación inicial docente" (OECD, 2015). Cabe destacar que la formación de profesores en Chile se encuentra en un momento de cambios importantes, "resta todavía realizar más investigación sobre esta temática (...) para mejorar los programas de acompañamiento de los estudiantes seleccionados y así fortalecer un tránsito exitoso a la carrera pedagógica" (González-García et al., 2017).

De acuerdo a lo expuesto, este artículo presenta un estudio realizado en el marco de un programa de Atracción de Talentos de Pedagogía con el objetivo de conocer y analizar la construcción de intereses vocacionales de jóvenes que participan en un programa propedéutico pedagógico en la Universidad Católica del Maule, Chile. Así, busca conocer los intereses vocacionales de los estudiantes que ingresan y se mantienen en el programa, con especial atención en aquellos estudiantes que muestran un aumento en el interés por estudiar pedagogía. Se trabajó con un diseño mixto explicativo secuencial (DEXPLIS) (Creswell y Plano, 2011). De los resultados se concluye que la participación de estudiantes en el programa impacta positivamente en sus intereses pedagógicos.

\section{Ingreso a las carreras de pedagogía en el contexto de América Latina}

En América Latina, los estudios indican que los docentes comparten una percepción de que su trabajo no es suficientemente valorado por la sociedad (Cox, et al., 2014). Este reconocimiento, anhelado por los profesores, ha impulsado a muchos países en la región a desarrollar formas de potenciar el estatus de la profesión docente, "se han desarrollado estrategias de estímulos, incentivos y premios con la doble finalidad de atraer y retener a los mejores docentes, y a la vez buscar que la sociedad vuelva a valorar la importancia de la profesión" (OECD, 2015). En el caso de Chile, se han incorporado cambios en las políticas educativas cuyo objetivo es el fortalecimiento de la calidad de la educación pública, de acuerdo a lo que contemplan los organismos internacionales y lo que muestra la evidencia científica, "el impulsor de las principales variaciones en el aprendizaje escolar es la calidad de los docentes" (Barber y Mourshed, 2018).

Desde esta perspectiva, se impulsa el desarrollo profesional del profesorado a través de la recuperación de la carrera docente (Bellei y Valenzuela, 2015). La transformación se proyecta de manera gradual a partir de la ley 20903, con el propósito de reconocer a la docencia, apoyar su ejercicio y aumentar su valoración para las nuevas generaciones (CPEIP, Mineduc, 2017). Al respecto, Said-Hung et al (2017) establece que "la agenda política actual busca romper la paradoja social existente, donde, pese al importante rol que tienen los docentes, esto no es reconocido socialmente o al menos a como se auto-perciben quienes se están formando. 
Entre los ámbitos que contempla la ley 20903, se define que solo universidades acreditadas pueden impartir carreras de pedagogía. De esta manera, se proyecta que la formación inicial docente aumente gradualmente sus exigencias de ingreso, al mismo tiempo, que incremente la calidad de sus programas de formación. Respecto a la vía de acceso a la carrera, la ley modifica el mecanismo de admisión a través de requisitos de postulación, los cuales buscan atraer a los estudiantes que realmente posean un interés por la docencia. Asimismo, se establece una nueva vía de ingreso que implica el haber cursado un programa de preparación y acceso a las pedagogías durante su enseñanza media. Estos programas deben tener la aprobación del Ministerio de Educación y además los estudiantes tienen la obligación de rendir la prueba de selección universitaria (Mineduc, 2016). Pese a ello, el puntaje no es determinante para el ingreso.

Como una manera de aportar al mejoramiento de la formación de los profesores de la Región del Maule, a través del acceso inclusivo y la permanencia de los estudiantes en las carreras de pedagogía, y en concordancia con la ley, la Universidad Católica del Maule implementa, desde el año 2014, el Programa de Atracción de Talentos en pedagogía. Este propedéutico se crea en el marco del Plan de Fortalecimiento Institucional en Formación Inicial de Profesores. Estos proyectos Mecesup constituyen plataformas para la instalación de las políticas públicas, a través de diversas iniciativas orientadas a la mejora en la formación docente. Estas propuestas buscan ser un aporte y, a la vez, una medida de regulación para las universidades formadoras de profesores. A nivel nacional, los propedéuticos de apoyo educativo y vocacional han sido una estrategia a la que, gradualmente, se han ido sumando las universidades para lograr un acceso más equitativo a la educación superior

\section{Contexto de investigación: Programa de Atracción de Talentos en Pedagogía}

Este programa se define como una estrategia de preparación a la universidad para escolares con mérito académico sobresaliente y, en el caso de la Universidad Católica del Maule, como vía de acceso inclusivo para estudiantes que pertenecen a los sectores más pobres de la región (Gajardo-Asbún y Schilling, 2019). El programa es inclusivo, ya que no discrimina por el puntaje de la prueba de selección universitaria. La normativa vigente exige un mínimo de puntaje que, en la gran mayoría de los casos, no es alcanzado por los estudiantes que provienen de los establecimientos municipales con los que colabora el programa. Al respecto, a nivel nacional, los estudios indican que un alto número de estudiantes de establecimientos con alta vulnerabilidad social y buen rendimiento escolar, obtiene bajos puntajes en la prueba de selección universitaria. Los que logran ingresar presentan problemas de adaptación socioemocional y académica (González et al., 2005).

Por tanto, la institución define que el propedéutico pedagógico tiene un importante valor social de inclusión, si se consideran las condiciones de desigualdad educativa que presentan los establecimientos escolares públicos de la Región. En consecuencia, el propedéutico pedagógico está dirigido a estudiantes de tercero y cuarto de enseñanza secundaria que se caracterizan como "talentosos" y que presentan interés por estudiar pedagogía. Ofrece a los jóvenes participar en procesos formativos conducentes a desarrollar un conjunto de habilidades académicas y personales que son necesarias para la integración a la vida universitaria, y considera como altamente relevante el fortalecer sus intereses vocacionales de ingreso. El funcionamiento del programa contempla que los jóvenes asistan a clases los días sábados, para participar en tres módulos de formación: habilidades comunicativas, habilidades de pensamiento lógico matemático y desarrollo personal y vocación pedagógica. En total, cada estudiante cursa tres semestres lectivos y, consecuentemente, la aprobación de estos cursos le permite ingresar directamente a cualquiera de las carreras de pedagogía que imparte la Universidad. Se reconoce que un sello distintivo de este programa, en la Universidad Católica de Maule, es la exploración de los intereses vocacionales de los jóvenes.

\section{Construcción de Intereses vocacionales}

El estudio de los intereses vocacionales presenta especial relevancia cuando se analizan las variables explicativas sobre la elección vocacional o profesional de un sujeto, por tanto, ha sido un campo dominado por la psicología y la orientación vocacional. En esta perspectiva, pueden ser definidos como las intenciones o aspiraciones a comprometerse en una dirección vocacional particular, las cuales aumentan la probabilidad de una acción de elección determinada (Cupani, 2006). De esta manera, permiten identificar las preferencias de una persona por cierta actividad y dan orientaciones para que esa actividad se desarrolle de mejor manera, o de manera satisfactoria.

Los intereses vocacionales poseen un rol protagónico en el análisis sobre la elección de una determinada carrera, han sido exhaustivamente examinados por los investigadores del comportamiento vocacional (Holland, 1997; Bergner et al., 2019; Nye et al., 2018), ya que, al ser estudiados de manera compleja, permiten predecir la elección de una carrera y la satisfacción en el ejercicio de esta. Al respecto, se podría discutir sobre cómo surgen estos intereses vocacionales, tal como Freire (1993) plantea, la vocación, en lugar de ser 
algo a priori de la historia, es por el contrario algo que viene constituyéndose en la historia. Por lo tanto, la trayectoria histórica del sujeto, y sus tramas sociales marcan la experiencia que influye en los intereses y proyecciones futuras. Lo anterior implica posicionarse desde el reconocimiento que los intereses vocacionales se construyen a partir de experiencias significativas en la vida de los jóvenes, amplían sus expectativas y orientan el proceso de toma de decisiones.

En el caso de los estudiantes que presentan interés por estudiar una carrera docente, el "proceso respondería a la construcción de una incipiente identidad orientada a la pedagogía, la cual es una combinación de interés o valoración por ser docente junto al desarrollo de capacidades que les permitan ingresar exitosamente a la carrera de pedagogía" (González-García, 2018). En definitiva, los propedéuticos constituyen espacios formativos que pueden enriquecer la experiencia de acercamiento a la docencia y la reflexión de los jóvenes sobre sus intereses vocacionales. A partir de lo anterior, este estudio desarrolló la siguiente hipótesis de investigación: El propedéutico Atracción de Talentos de pedagogía de la UCM acrecienta el interés vocacional que presentan los jóvenes de enseñanza media por la pedagogía.

\section{METODOLOGÍA}

El estudio se realizó con un diseño mixto explicativo secuencial, caracterizado por una primera fase de análisis de datos cuantitativos, seguida de otra en la que se recogen y evalúan datos cualitativos. Este diseño favorece que los resultados de la segunda etapa se construyan a partir de los resultados de la primera. De esta forma, los resultados cualitativos aportan y profundizan la interpretación y explicación de los descubrimientos cuantitativos iniciales (Creswell y Plano, 2011.) Asimismo, los resultados cuantitativos sirven para orientar la definición de la muestra en coherencia con los propósitos que se busca alcanzar.

\section{Participantes:}

Se consideró un muestreo no probabilístico, dado que los participantes se seleccionaron de forma intencionada a través de criterios de investigación, a saber: a) ser estudiante de enseñanza media de la Región del Maule; b) participar regularmente de las actividades formativas del propedéutico pedagógico en la UCM; c) presentar un incremento positivo en el interés vocacional por la pedagogía (diferencia pre y pos test); d) participación voluntaria en el estudio. En la etapa cuantitativa se trabajó con el total de estudiantes que ingresaron al programa (20) y luego, en la segunda etapa, el interés se focalizó en aquellos jóvenes que presentaron resultados positivos en el test de intereses vocacionales. En total fueron seis estudiantes que avanzaron a la fase cualitativa del estudio.

\section{Procedimientos:}

Contempló la aplicación del Inventario de intereses Ramírez Vera para la identificación de áreas de proyección vocacional (Ramírez, 2013), instrumento que mide la detección de áreas de proyección vocacional. Cabe señalar que el propedéutico aplica regularmente este instrumento al momento que los jóvenes ingresan. El test expone 180 tareas o actividades frente a las cuales el estudiante puede o no mostrar su interés o agrado. Las áreas de proyección vocacional contemplan dieciocho factores como profesiones u oficios: comerciales y financieros, agrícolas y pecuarios, ecológicos y medioambientales, religiosos y espirituales, militares, deportivos y recreativos, científico-sociales, científico-naturales, matemáticos, artístico-visuales, artísticoliterarios y de representación, artístico-musicales, informáticos y virtuales, mecánicos y técnicos, pedagógicos y educativos, manuales y artesanales, de compromiso político y social, comunicacionales. Este inventario ha sido validado previamente con un alpha de Cronbach de 0.91. Asimismo, el índice de adecuación muestral de KMO resultó bueno (.82), así como la prueba de esfericidad de Bartlett, $X 2(730.43)=1931.52 ; p<.000$. El análisis sicométrico concluye que la validez de constructo de la escala es adecuada para inducir los factores mencionados anteriormente. Este test tiene la particularidad que incorpora el concepto de intereses transversales (Ramírez y Sanhueza, 2015).

Para esta investigación, el pre test se aplicó en abril del 2017. A continuación, el programa contempló la participación de los jóvenes en una serie de actividades motivacionales/vocacionales y de formación académica desarrolladas en la Universidad, durante el periodo lectivo de cuatro meses consecutivos. Los módulos cursados fueron: a) Habilidades comunicativas, orientado a desarrollar destrezas y capacidades vinculadas con la comprensión y producción de textos orales y escritos, potenciar la oralidad y ampliar las referencias culturales de base; b) Habilidades de pensamiento lógico-matemático, cuyo propósito es desarrollar destrezas y capacidades vinculadas con la promoción del pensamiento lógico matemático y la resolución de problemas; c) Desarrollo personal y vocación pedagógica, orientado a la promoción y desarrollo de habilidades, destrezas y recursos personales, tales como, autoconocimiento, resiliencia, comunicación, compromiso y desarrollo integral. Posteriormente, se aplicó nuevamente el instrumento (post test). Finalmente, los datos fueron tabulados según tendencias en las áreas de interés vocacional por la pedagogía. 
En cuanto al análisis de los datos cuantitativos, se procedió a verificar la normalidad través del, test "Shapiro Wilks", que permitió establecer con mayor precisión qué estudiantes aumentaron el interés por estudiar pedagogía, en el marco del programa de atracción de talentos. Luego de este análisis, se seleccionaron seis sujetos que avanzaron a la etapa cualitativa. La fase cualitativa de la investigación utilizó la entrevista semi estructurada como técnica de recolección de datos. Se aplicó una entrevista por sujeto, con la finalidad de profundizar en los significados que los jóvenes atribuyen a sus experiencias de formación, y reconocer aquellas que han marcado su interés por estudiar pedagogía en el marco del programa. Para asegurar los requisitos éticos de la investigación, se solicitó la firma de un consentimiento informado, antes de aceptar la participación en la entrevista.

Con relación al análisis cualitativo, se utilizó el microanálisis de datos propuesto por Strauss y Corbin (2002). A partir de la codificación de las entrevistas se formularon categorías inductivas (Mayring, 2002). Se identificaron las siguientes categorías: i) participación en programa PAT, ii) aproximaciones a la docencia y iii) reflexiones sobre el interés por estudiar pedagogía.

\section{RESULTADOS}

Los resultados se presentan en dos niveles de análisis. La primera fase, cuantitativa, presenta los resultados del pre y post test aplicado a los 20 estudiantes del programa. La segunda fase, cualitativa, detalla las categorías de análisis levantadas a partir de las entrevistas focalizadas en los seis estudiantes que aumentaron su interés por estudiar pedagogía durante su participación en el programa.

\section{Identificación de intereses vocacionales en el marco del programa de atracción de talentos}

Para establecer si existen diferencias estadísticamente significativas entre los valores promedio de cada test de intereses vocacionales, se procedió a verificar la normalidad de los datos, utilizando el test "Shapiro Wilks", en que la hipótesis nula señala: "Los datos provienen de una distribución normal". El valor $p$ asociado a esta fue 0,085 . Los valores $p$ menores a 0,05 indican que existe evidencia para rechazar la hipótesis nula, es decir, los datos provienen de una distribución normal. Dado que los datos poseen una distribución normal, se utilizará un test t pareado, para verificar si existen diferencias entre los dos grupos. La hipótesis nula asociada a la prueba es: "Al menos uno de los grupos presenta diferencias significativas". El valor asociado a esta prueba es de 0,018. Lo cual indica que existe evidencia para rechazar la hipótesis nula. Es decir, uno de los grupos es mayor al otro, en este caso se confirma la hipótesis de investigación que establece que la participación de los estudiantes en el programa incrementa el interés vocacional por la pedagogía.

La Figura 1 expone los cambios en los intereses pedagógicos de los 20 estudiantes que participan en el programa durante el año 2016.

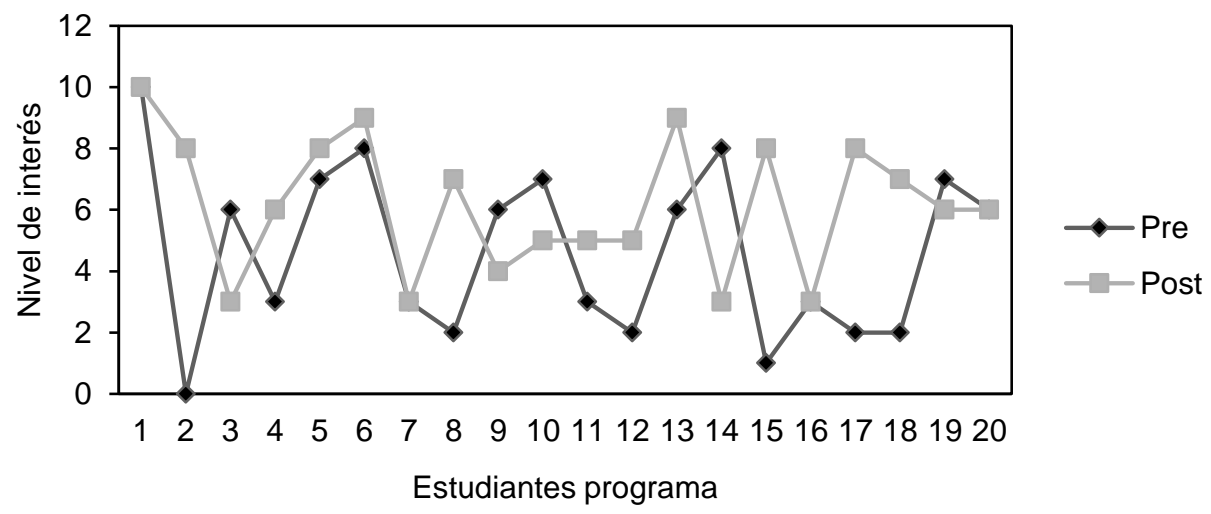

Fig.1: Resultados pre y post test por estudiante del programa.

Se aprecia, a partir de la figura 1, que, de los 20 estudiantes, 14 presentaron un aumento en el interés por la pedagogía durante su participación en el programa, lo que representa un $70 \%$ de la muestra; 3 de ellos, es decir el 15\% mantuvieron el mismo nivel de interés y, finalmente, 3 estudiantes disminuyeron su interés, lo que simboliza el $15 \%$ del total. Por lo tanto, es posible reconocer un efecto positivo del programa respecto a la definición de intereses pedagógicos.

\section{Elementos que influyen en la construcción del interés vocacional por la pedagogía}

A partir de los resultados cuantitativos, que muestran los efectos positivos del programa, surge el interés por conocer ¿cuáles son los significados que los jóvenes han construido durante su experiencia en el 
propedéutico? ¿Cuáles consideran como factores positivos en la construcción de su interés vocacional? Con el objetivo de dar respuesta a estas preguntas, se presentará el análisis de las entrevistas a través de categorías y sub categorías.

\section{Participación en Programa de Atracción de Talentos en Pedagogía}

Esta categoría permite develar los significados de los jóvenes en relación a su participación en el propedéutico. Específicamente, sobre los factores que estarían favoreciendo los intereses vocacionales por la pedagogía. Se identifican dos sub categorías: 1) actividades formativas del programa; 2) experiencias significativas con profesores invitados. La primera sub categoría hace referencia a la percepción de los estudiantes frente a su participación en diversas actividades que ofrece el programa, entre las cuales destacan los espacios de diálogo y desarrollo personal que enriquecen la reflexión sobre el rol docente en la sociedad actual. Estas actividades se seleccionan reconociendo que "al desarrollar una actividad, es importante tener en cuenta los factores motivacionales del estudiante, ya que pueden afectar el ambiente académico en el que se encuentra" (Sánchez et al., 2016). La segunda sub categoría muestra el valor que atribuyen a los profesores que han participado en el programa. Específicamente, en la relación pedagógica establecida al compartir sus experiencias de vida. Estos relatos de experiencias profesionales contribuyen a reconocer la identidad y rol histórico que tiene la docencia, por tanto, "se desarrolla de manera que refleja la combinación única de cultura, contextos y experiencias que poseen" (Edwards y Edwards, 2016). La Tabla 1 presenta algunos fragmentos de las respuestas relacionadas con esta dimensión.

Tabla 1: Fragmentos de respuestas de los jóvenes relacionadas a la categoría: Participación en Programa de Atracción de Talentos en Pedagogía

\begin{tabular}{|c|c|}
\hline Actividades formativas del programa & Experiencias significativas con profesores invitados \\
\hline $\begin{array}{l}\text { “... a través del PAT me he dado cuenta que Pedagogía } \\
\text { es una carrera que me gusta mucho..." (E2) } \\
\text { “...el profesor Héctor y el profesor Alejandro me } \\
\text { motivaban mucho, porque yo veía que ellos sí tienen } \\
\text { vocación para enseñar...con ellos yo conocí que } \\
\text { pedagogía era una carrera súper linda..." (E4) } \\
\text { “...Aquí los profesores son diferentes (...) en la forma de } \\
\text { explicar las cosas, son amorosos y siempre me están } \\
\text { contando experiencias y esas cosas que a uno le da más } \\
\text { interés por estudiar pedagogía..." (E6) } \\
\text { “...me motivaba mucho, porque yo veía que ellos sí } \\
\text { tienen vocación para enseñar, yo veo a todos los } \\
\text { profesores de acá que nos cuentan lo bueno y lo malo, } \\
\text { entonces es como más transparente y veo que todos han } \\
\text { tenido experiencias lindas y que es muy linda la carrera } \\
\text { en sí." (E5) }\end{array}$ & $\begin{array}{l}\text { "...ellos muestran como su realidad (...) como eso, le ha } \\
\text { ayudado en su vida, entonces como que nunca ser profesor } \\
\text { fue una carga, sino más bien una experiencia que es } \\
\text { bastante bonita, me gusta escuchar las historias porque } \\
\text { siempre sacan algo bueno, siempre es enriquecedora la } \\
\text { vida..." (E2) } \\
\text { "... los profesores nos transmitieron perseverancia, el amor } \\
\text { a hacer lo que le gusta y que no importa las cosas pero sí } \\
\text { seguir adelante, amor a la profesión..." (E3) } \\
\text { "...cuando vienen profesores que ya están jubilados, } \\
\text { cuentan que cuando ellos estudiaban en partes de campo } \\
\text { tenían que atravesar largos caminos, los niños no iban } \\
\text { uniformados como ahora, las escuelas eran chiquititas, } \\
\text { pero aun así ellos hacían sus clases..." (E5) } \\
\text { "...lo sentían con pasión, era más que explicar, era como } \\
\text { que se sentían hasta orgullosos de eso y me gustaba } \\
\text { observarlo..." (E6) }\end{array}$ \\
\hline
\end{tabular}

Se evidencia que la participación de los estudiantes en el programa, y específicamente los espacios de diálogo con sus docentes han sido altamente relevantes para conocer la carrera docente y el valor de la experiencia vivida con pasión por la educación. Dentro de dichas actividades, los estudiantes destacan la interacción con sus profesores en los módulos formativos. Estos docentes tienen la particularidad de ser profesores con experiencia en el sistema escolar, además de trabajar en la universidad. Asimismo, los jóvenes reconocen que los docentes con trayectoria destacada (profesores jubilados invitados regularmente al programa) les han permitido enriquecer su percepción sobre la docencia. Estos diálogos contribuyen a conocer diversas miradas y contextos de la educación nacional, aproximándose a reflexiones orientadas a la vocación por la docencia. Este podría ser un factor que fortalece el interés vocacional, pues permite al estudiante descubrir experiencias que contribuyen a reafirmar su decisión por continuar estudios de pedagogía.

\section{Aproximaciones a la docencia}

Esta categoría detalla los significados que explicitan los jóvenes respecto a sus experiencias previas de socialización con la docencia en el marco de sus entornos sociales. Se reconocen dos sub categorías: 1) enseñanza entre pares; 2) participación en trabajo comunitario. La sub categoría, enseñanza entre pares, hace referencia a las experiencias de formación en que los estudiantes han sido protagonistas apoyando el aprendizaje de sus compañeros o familiares. La sub categoría, participación en trabajo comunitario, devela que los estudiantes reconocen que la experiencia de participación voluntaria en trabajo social ha fortalecido la vocación de servicio. Esta experiencia, al no ser formal u obligatoria, adquiere particular relevancia en el interés por la pedagogía. En consecuencia, "la discusión constructiva con los pares agrupados de acuerdo con una relativa heterogeneidad puede llevar a los participantes a revisar, ajustar o ampliar sus pensamientos, 
tan necesario como sea posible para lograr que sus ideas sean entendidas por los demás" (Olave y Villarreal, 2014). Por tanto, es considerada como uno de los factores que fortalece el proceso decisional por el interés pedagógico. Iniciativas como aprendizaje desde la propia comunidad, de líderes en scout, pastoral, deportes, aportan en la construcción de intereses a un nivel más colectivo y experiencial, dado que "la integración de proyectos comunitarios muestra el trabajo interdisciplinario y cómo cada disciplina fortalece a la otra" (Burrows et al., 2018), por lo tanto, enriquece el sentido colectivo de los estudiantes a partir de los problemas y desafíos reales. La Tabla 2 presenta algunos fragmentos de las respuestas relacionadas con esta dimensión.

Tabla 2: Fragmentos de respuestas de los jóvenes relacionadas a la categoría aproximaciones a la docencia

\begin{tabular}{|c|c|}
\hline Enseñanza entre pares & Participación en trabajo comunitario \\
\hline $\begin{array}{l}\text { “...le enseño también a mis compañeras y me quedo con } \\
\text { tres chiquillas cuando tengo tiempo, los martes y jueves, } \\
\text { sola a enseñarles..." (E1) } \\
\text { “...Yo cuando era chiquitita, así como de primer ciclo, me } \\
\text { gustaba mucho lo que era enseñarle a mis compañeros } \\
\text { porque yo no hice ni kínder ni pre kínder, pero sí mi } \\
\text { mamá me enseñó al tiro, desde antes que entrara al } \\
\text { colegio (...) mientras a mis compañeros les costaba } \\
\text { juntar letras, me gustaba mucho enseñarles todo lo que } \\
\text { yo tenía adelantado..." (E5) } \\
\text { "... Tengo un hermano más pequeñito y también ha sido } \\
\text { importante para decidir esto de la pedagogía porque yo } \\
\text { le enseño cosas, y además estoy interna y siempre } \\
\text { estudio en grupo, entonces es como enseñarles a mis } \\
\text { compañeras, también me hace darme cuenta que puedo } \\
\text { ser capaz de enseñarle a alguien, porque a ellas les va } \\
\text { bien...." (E6) }\end{array}$ & $\begin{array}{l}\text { "...en primero medio ya entré con todo al centro de alumnos } \\
\text { de la enseñanza media, y me metía a todo lo que eran } \\
\text { actividades pastorales juveniles y la infancia misionera. En } \\
\text { infancia misionera trabajábamos con } 50 \text { a } 60 \text { niños, éramos } \\
\text { seis monitoras, toda mis amigas y una asesora quién era la } \\
\text { que nos acompañaba, siento que ahí me aproximé al ser } \\
\text { profesor..." (E1) } \\
\text { "...Yo creo que sí, de cierta forma han influido (...) no sé, es } \\
\text { que no soy muy buena explicando, pero me gusta ayudar, } \\
\text { quiero algo en lo que pueda ser útil para los demás..." (E5) }\end{array}$ \\
\hline
\end{tabular}

De acuerdo a lo señalado en la tabla 2, se puede evidenciar que la sub categoría, enseñanza entre pares, también aparece como experiencia significativa en la construcción del interés vocacional, dado que permite desarrollar el gusto o placer por enseñar, donde: "me gusta explicarles a mis compañeros", "me gustaba mucho enseñarles" es la frase que más se repite. Por lo tanto, estas primeras aproximaciones de enseñanza, servicio y trabajo colectivo marcan el interés vocacional de los jóvenes.

\section{Reflexiones sobre el interés por estudiar pedagogía}

Esta categoría detalla las reflexiones de los jóvenes en relación a lo que ha significado decidir que quieren estudiar pedagogía. Estas reflexiones aportan a la ampliación del sentido sobre su proyección vocacional. De esta categoría surgen tres sub categorías: 1) intereses personales por las disciplinas pedagógicas; 2) proyecciones como docentes; 3 ) apoyo familiar.

La sub categoría, intereses personales, hace referencia a una inclinación por los gustos personales que orientan a los jóvenes a elegir una carrera universitaria o especialidad, como señala Shirai et al. (2013) "la inclinación hacia los intereses personales de desarrollo se relaciona positivamente con la intención de un futuro laboral, por lo tanto, los intereses personales van ligados a lo que cada individuo quiere desempeñar en un futuro". La sub categoría, proyecciones como docente, tiene relación con cómo los estudiantes visualizan su futuro. Estas proyecciones no se dan en el vacío, los estudiantes no están aislados del contexto en el cual desarrollan sus expectativas, los condicionantes sociales influyen en la mirada del futuro. Transitar por el sistema educativo ya no representa garantía de movilidad social ascendente como lo pensaban los sectores medios. Tampoco garantiza una mejor inserción laboral, pero la educación sí sigue siendo el medio necesario para acceder a un trabajo (Corica, 2012). Al respecto, se puede apreciar que sus características sociales de origen condicionan la auto percepción y las proyecciones futuras de auto realización. Asimismo, queda en evidencia que los estudiantes entrevistados proyectan la posibilidad de retornar a sus realidades de origen para aportar al desarrollo de su comunidad.

La sub categoría, apoyo familiar, se define por el soporte y acompañamiento que da la familia al estudiante que participa del propedéutico. Este hallazgo es consistente con el resultado de otras investigaciones que reconocen que uno de los factores que influye en que un joven concluya exitosamente una licenciatura o programa de estudios se relaciona con el apoyo que recibe por parte de la familia, lo que reafirma el importante papel que juega dicha institución en todo proceso formativo (Abarca et al., 2015). Además, la identificación con sus progenitores podría impactar directamente en la elección vocacional (Rivas 1998). Si se considera el perfil del estudiante que ingresa al programa, en su mayoría primera generación en acceder a la universidad, pertenecientes a zonas rurales y en algunos casos aislados de la Región, se debe reconocer que su participación en el programa de acceso inclusivo puede significar cumplir el sueño de movilidad social de su familia. 
Tabla 3: Fragmentos de respuestas de los jóvenes relacionadas a la categoría reflexiones sobre docencia.

\begin{tabular}{|c|c|c|}
\hline Intereses personales de desarrollo & Proyecciones como docentes & Apoyo familiar \\
\hline $\begin{array}{l}\text { "...la ciencia es mi vida, me encanta, } \\
\text { no hay nada más que me guste ..." } \\
\text { (E2) } \\
\text { "...me gusta enseñar..." (E3) } \\
\text { "...mi personalidad puede ir más allá } \\
\text { y con el programa se me sigue } \\
\text { viendo eso, ya no me da tanta } \\
\text { vergüenza conversar con más } \\
\text { personas..." (E4) } \\
\text { “...me ha ayudado también a } \\
\text { expresarme mucho más, porque a } \\
\text { mí me ha costado un poquito y yo les } \\
\text { decía tal vez yo no sirva para esto, } \\
\text { pero aquí se aprende..." (E5) }\end{array}$ & $\begin{array}{l}\text { “...siento que la pedagogía se mete } \\
\text { en la persona, uno siempre va a ser } \\
\text { profe y eso es lo que quiero ser. En la } \\
\text { calle jhola profe! ¿Cómo está? Y yo } \\
\text { misma saludo a los profes cada vez } \\
\text { que los veo, y les digo profe, no les voy } \\
\text { a decir por su nombre, porque son } \\
\text { reconocidos como tales..." (E1) } \\
\text { “...si veo a un niño que está triste, ver } \\
\text { qué le pasa, ver cómo está, jvamos } \\
\text { que se puede! Seguir la relación con el } \\
\text { estudiante es muy importante..." (E3) } \\
\text { “...a mí me gustaría ejercer mi } \\
\text { profesión allá en mi comunidad...” } \\
\text { (E4) }\end{array}$ & $\begin{array}{l}\text { "... han habido días en los que yo no } \\
\text { quiero seguir y estoy cansada, ellos } \\
\text { me dicen sigue adelante, tú puedes, } \\
\text { ellos han sido uno de los pilares } \\
\text { fundamentales para mí..." (E3) } \\
\text { “...Fue positivo, no se negaron en } \\
\text { decirme cómo se te ocurre estudiar } \\
\text { esto, más bien estuvieron muy } \\
\text { dispuestos a ayudarme..." (E6) }\end{array}$ \\
\hline
\end{tabular}

En relación a la tabla 3, se puede señalar que la sub categoría, intereses personales, hace referencia a los gustos por las distintas disciplinas pedagógicas. Asimismo, en su interés por mejorar aspectos personales en los cuales perciben que están descendidos. Por otra parte, la sub categoría, proyecciones como docentes, expone la capacidad de visualizar su futuro profesional, a través del trabajo en sus comunidades. Algunos estudiantes mencionan que les gustaría trabajar en el colegio donde estudiaron, dando a entender que sus experiencias escolares han sido relevantes y se expresan en el compromiso social con su comunidad. En sus reflexiones plantean que se visualizan como profesores preocupados de sus estudiantes no solo en el ámbito académico, sino también en lo personal. Finalmente, la sub categoría apoyo familiar se presenta como un pilar para la participación de los jóvenes en el programa, ya que manifiestan que los padres son un soporte fundamental para sus decisiones, preferencias y momentos difíciles y especialmente, en la aspiración de continuar estudios superiores en el ámbito de la pedagogía.

\section{DISCUSIÓN DE LOS RESULTADOS}

A partir de los resultados cuantitativos y cualitativos se puede establecer que la participación de los jóvenes en el propedéutico pedagógico incrementa positivamente el interés vocacional por la pedagogía. Este cambio se atribuye a una serie de factores explicativos, presentes en el marco del programa. Los resultados ponen de manifiesto que el interés vocacional de los jóvenes responde a un proceso de exploración y autoconocimiento que se desarrolla en relaciones de dialogo y reconocimiento con docentes que poseen experiencia pedagógica y compromiso con su profesión. Por lo tanto, este hallazgo es coincidente con lo evidenciado en investigaciones previas que señalan que se estaría fortaleciendo la construcción de una incipiente identidad orientada a la pedagogía a partir de la valoración a la profesión docente (González-García, 2018).

El análisis cualitativo devela que los estudiantes responden favorablemente a los desafíos formativos que ofrece el programa, con especial valoración de las experiencias formativas en la cuales prevalece el diálogo y la reflexión colectiva durante el proceso de aprendizaje, conectándolos con referentes sociales significativos como son los docentes con trayectorias profesionales destacadas, docentes que ya se encuentran jubilados y sus profesores de los módulos. Este diálogo, en que se releva la experiencia vivida y los saberes docentes, aporta a la construcción de significados sociales que favorecen la comprensión histórica del rol docente en la sociedad, motivando a que los estudiantes conecten su propia experiencia y acciones con lo que Freire (1993) define como la creación y recreación del conocimiento comúnmente compartido. Asimismo, destaca el reconocimiento al compromiso de servicio que han desarrollado los docentes en nuestra Región. De esta forma el programa les muestra el mundo real, no idealizado sobre la docencia, y los motiva a fortalecer sus decisiones vocacionales.

Asimismo, los jóvenes reconocen que los primeros acercamientos a la docencia se presentan tempranamente, en experiencias cotidianas que los vinculan a la enseñanza entre pares. Especialmente con sus familiares cercanos y compañeros en la escuela. Como en su mayoría han sido estudiantes aventajados, asumen la responsabilidad de apoyar a sus pares. La satisfacción alcanzada en estas experiencias formativas propicia una sensación de logro y de servicio donde reconocen inicialmente su interés vocacional. Esto concuerda con la evidencia de otros estudios que plantean que "los factores motivacionales predominantes en los futuros docentes se enmarcan en argumentos de tipo intrínsecos y altruistas" (Said-Hung et.al., 2017). Consecuentemente, el propedéutico, al ofrecer un espacio formativo que los conecta con la reflexión sobre esas experiencias iniciales, se convierte en un factor positivo para la construcción del interés pedagógico. 
Los estudiantes reconocen que un factor que explica su permanencia en el programa y la reafirmación vocacional, es que sus decisiones son acompañadas e incentivadas desde el apoyo de sus familias. Esto proyecta una alta expectativa sobre su ingreso a la carrera de pedagogía.

\section{CONCLUSIONES}

La evidencia muestra que el propedéutico aporta al fortalecimiento de los intereses vocacionales de los jóvenes por su propuesta pedagógica centrada en el diálogo y el reconocimiento. La formación del programa no solo prepara a los jóvenes en un sentido funcional de "adaptación académica a la universidad", sino que reconoce sus intereses, condiciones de vida, y la historia personal que los lleva a seleccionar la carrera de pedagogía como una opción vocacional.

Los jóvenes que ingresan al programa reconocen como altamente relevante el espacio de reflexión colectiva con referentes sociales significativos (profesores en ejercicio y con trayectoria destacada), que ofrece la posibilidad de conectar sus intereses vocacionales con los saberes docentes e historias de vida de profesores comprometidos con la educación de la Región. Por consiguiente, el programa se convierte en un espacio de reconocimiento que potencia la participación de los jóvenes como agentes de cambio social.

Otro hallazgo es la valoración que otorgan los jóvenes a las experiencias de trabajo comunitario. Considerando sus experiencias previas, y también aquellas instancias colectivas que se generan en el marco del propedéutico. Al reconocer el servicio hacia su comunidad como parte importante de la labor del profesor, surge un interés de compromiso político y social. Esto queda en evidencia cuando proyectan en su futuro laboral retornar a sus territorios de origen para contribuir al desarrollo de sus comunidades.

\section{REFERENCIAS}

Abarca, M. S., M. Gómez y M. Covarrubias, Análisis de los factores que contribuyen al éxito académico en estudiantes universitarios, ISSN: 2255-453X, Revista Internacional de Educación y Aprendizaje, 3(2), 124-137 (2015)

Barber, M. y M. Mourshed, How the World's Best-Performing School Systems Come out on Top, ISSN: 0718-6002 McKinsey y Company PREAL (2007)

Bellei, C. y J. Valenzuela, El estatus de la profesión docente en Chile. Percepción de los profesores acerca del estatus profesional de la docencia. Centro de Investigación Avanzada en Educación Universidad de Chile Núcleo Milenio en Ciencias Sociales, La Profesión Docente en Chile, 1-32 (2015)

Bergner, S., A. Kanape y R. Rybnicek, Taking an interest in taking the lead: The influence of vocational interests, leadership experience and success on the motivation to lead, doi:10.1111/apps.12150, Applied Psychology, 68(1), 202-219 (2019)

Burrows, A., M. Lockwood y otros tres autores, Integrated STEM: Focus on Informal Education and Community Collaboration through Engineering, doi:10.3390/educsci8010004, Education Sciences, 8(1), 4, 1-15 (2018)

Corbin, J. y A. Strauss, Basics of qualitative research: Techniques and procedures for developing grounded theory (2008)

Corica, A., Las expectativas sobre el futuro educativo y laboral de jóvenes de la escuela secundaria: Entre lo posible y lo deseable, doi:10.4067/S0718-22362012000100004, Última Década, 36, 71-95 (2012)

Cox, C., C. Beca y M. Cerri, Docentes para una educación de calidad en América Latina: problemas y orientaciones de políticas, ISBN: 978-607-96349-1-9, Realidades y Prospectiva Educativa, Tomo I, 41-92 (2014)

CPEIP-MINEDUC, Orientaciones Sistema de Desarrollo Profesional Docente, 1-53 (2017)

Creswell, J. y V. Plano Clark, Designing and Conducting Mixed Methods Research, Thousand Oaks, ISBN10: 1412975174 CA: Sage publications, inc.,(2011)

Cupani, M. y E. Pérez, Metas de elección de carrera: Contribución de los intereses vocacionales, la autoeficacia y los rasgos de personalidad, ISSN: 0325-8203, Interdisciplinaria, 23 (1), 81-100 (2006)

Edwards, F. y R. Edwards, A story of culture and teaching: the complexity of teacher identity formation, doi:10.1080/09585176.2016.1232200, Curriculum Journal, 28(2), 190-211 (2016)

Freire, P., Pedagogía de la esperanza: un encuentro con pedagogía del oprimido, México, Siglo XXI (1993)

Gajardo-Asbún, K. y C. Schilling, Talent Attraction Program in Pedagogy to Promote Pedagogical Vocation among Young People in the Maule Region, Research in Education. Teacher Training Issues, Nova Science Publishe, 107 -120, New York, USA (2019)

González L., D. Uribe y S. González, Estudio sobre la repitencia y deserción en la educación superior chilena digital observatory for higher education in latin america and the caribbean, Unesco (2005)

González-García, G., Criterios de justicia social para atraer buenos postulantes de liceos públicos a la carrera pedagógica. Políticas educativas y su impacto en las comunidades, investigación en educación para la justicia social, 1를., Ediciones Universidad Católica del Maule, Talca, Chile, 189-208 (2018) 
González-García, G., W. Walker, R. García y C. Abarca, Hacia una construcción teórica y contextualizada del talento escolar para la pedagogía en liceos públicos. Investigación para la formación de profesores. Aportes desde la Universidad Católica Silva Henríquez, 1aㅡ Ed., Ediciones UCSH, 33-52, Santiago, Chile (2017)

Holland, J., Making vocational choices: A theory of vocational personalities and work environments, Psychological Assessment Resources, 3를., (1997)

Kvale, S., Interviews: An introduction to qualitave research interviewing, Thousand Oaks, CA, Sage (1996)

Lincoln, Y., S. Lynham y E. Guba, Paradigmatic controversies, contradictions, and emerging confluences, revisited. En N. K. Denzin y Y. S. Lincoln (Eds.), The Sage Handbook of Qualitative Research, 97-128 (2011)

Mayring, P., Qualitative Content Analysis, doi: 10.17169/fqs-1.2.1089, Forum Qualitative Sozialforschung / Forum: Qualitative Social Research, 1(2), Art. 20 (2000)

MINEDUC, Biblioteca del Congreso Nacional de Chile - www.leychile.cl - documento generado el 13-Jun-2012 (2016)

OECD, Education in Chile, Reviews of National Policies for Education, doi:10.1787/9789264284425-OECD, Publishing, Paris (2017)

OECD, Reformulando la carrera docente en Chile. Evidencia Internacional Seleccionada, OECD (2015)

Olave, I. y A. Villarreal, El proceso de corregulación del aprendizaje y la interacción entre pares, ISSN: 1405-6666, Revista Mexicana de Investigación Educativa, 19(61), 377-399 (2014)

Ramírez, L. y S. Sanhueza, Diseño y validación de un inventario de intereses para identificar áreas de proyección vocacional (IPV) en estudiantes de educación secundaria, ISSN: 0719-9872, 48, 55-84 (2015)

Ramírez, L., Inventario de intereses Ramírez Vera para la identificación de áreas de proyección vocacional, ISSN: 0717 2656 (2013)

Rivas, F., Psicología vocacional: Enfoques del asesoramiento, 3를., ISBN: 9788471123213, Madrid, Morata (1998)

Said, E., G. Gratacós y J. Cobos, Factors affecting the choice of teaching programs in Colombia, doi:10.1590/s1517970220170116097, Educação e Pesquisa, 43(1), 31-48 (2017)

Sánchez, E.J., J.N. Hooker y A. Carrillo-Ramos, Motivacionales de los Estudiantes Motivational Factors, doi:10.1109/ColumbianCC.2016.7750780, Institute of Electrical and Electronics Engineers Inc. Conference Paper, 1-8 (2016)

Shirai, T., H. Shimomura, T. Kawasaki y otros dos autores, Job search motivation of part-time or unemployed Japanese college graduates. doi:10.1007/s10775-013-9241-3, International Journal for Educational and Vocational Guidance, 13(2), 95-114 (2013)

Vaillant, D., El fortalecimiento del desarrollo profesional docente: una mirada desde Latinoamérica, doi.org/10.15366/jospoe2016.5, Journal of supranational policies of education, 5, 5-21 (2016) 